\title{
The Effect of an Er,Cr:YSGG Laser in the Management of Intrabony Defects Associated with Chronic Periodontitis Using Minimally Invasive Closed Flap Surgery. A Case Series
}

\author{
Rana Al-Falaki ${ }^{1}$, Francis Hughes ${ }^{2}$, Reena Wadia ${ }^{2}$, Christie Eastman ${ }^{3}$, \\ Elias Kontogiorgos ${ }^{4}$, Samuel Low ${ }^{3}$ \\ 1: Private Practice, London, UK. \\ 2: Dept of Periodontology, Kings College London Dental Institute, University of London \\ 3: Department of Periodontology, University of Florida, College of Dentistry \\ 4: Department of Restorative Sciences, Baylor College of Dentistry, Texas A\&M University
}

\begin{abstract}
Aims: This is an extended case series of patients treated with an Erbium, Chromium: Yttrium Scandium Gallium Garnet (Er,Cr:YSGG) laser as an adjunct to scaling for the management of intrabony defects.

Materials \& methods: 46 patients with 79 angular intrabony defects associated with pocket depths of $>5 \mathrm{~mm}$, and a mean age of $53 \pm 9$ years presenting with chronic periodontitis were included in the analysis. All patients underwent a localized minimally invasive closed flap surgery utilizing Er,Cr:YSGG laser therapy. Final radiographs and pocket depths were compared to pretreatment measurements with a time period of $8 \pm 3$ months.

Results: Treatment resulted in significant overall pocket depth reduction. The mean pre-op probing depth was $8.1 \pm 1.9 \mathrm{~mm}$, reducing to $2.4 \pm 0.9 \mathrm{~mm}$ post-treatment. Bony infill of the defects was visible radiographically and there was an increase in overall radiographic coronal osseous height compared to a pre-treatment baseline. Radiographs of 15 of the defects were available for further measurements after $>12$ months, and showed in these sites there was a significant reduction in intrabony defect depth, but no change in suprabony bone height. 9 of the 15 sites showed 50\% or more, bony infill of the intrabony defect.

Conclusions: The results demonstrate that the utilization of an Er,Cr:YSGG laser in a closed flap approach with chronic periodontitis may be of significant clinical benefit. Further studies using this laser surgical protocol are required to test these observations in well-designed randomized controlled trials.
\end{abstract}

Key words: Er,Cr:YSGG laser • root surface debridement • intrabony defects • bone growth • pocket reduction

\section{Introduction}

Surgical treatment for the management of periodontal pockets associated with intrabony defects can be challenging. Due to access, these defects often require surgical intervention, employing regenerative surgical

\section{Addressee for Correspondence:}

Dr Rana Al-Falaki BDS, MFDS RCSI, MClinDent, MRD RCS (Eng) Specialist In Periodontics

Al-Fa Perio Clinic, 48A Queens Road, Buckhurst Hill, Essex IG9 5BY

Tel: +44 2085060701 e-mail: info@al-faperio.co.uk

C2016 JMLL, Tokyo, Japan techniques including barrier membranes, osseous graft material or application of a biologically active material. The alternative therapy is root surface debridement, which may have an unpredictable outcome with the exception of narrow three wall bony defects.

There has been increasing interest in the use of lasers for managing periodontitis including non-surgical root surface debridement and a range of surgical therapies, with minimal clinical trials to demonstrate

Received date: February 2nd, 2016 Accepted date: June 6th, 2016 
efficacy. The Nd:YAG has shown improvement in periodontal clinical trials with clinical parameters including a histologic assessment. 1, 2, 3). The Er:YAG laser has been proposed as an alternative or adjunct to conventional mechanical periodontal therapy. Randomized clinical trials of these wavelengths for managing periodontitis have shown mixed advantages over conventional flap treatment. $4,5,6,7)$ There are few studies that have evaluated the use of the Er,Cr:YSGG watercooled laser 8,9 ).

The objective of this case series was to report on, and develop a retrospective analysis of the effectiveness of utilizing an Er,Cr:YSGG laser as a minimally invasive surgery procedure to manage periodontitis associated with intrabony defects.

\section{Materials and methods}

Forty six patients (31 females and 15 males) with an age range of 35 to 76 and a mean age of 53 years, with a clinical diagnosis of localized or generalized moderate to advanced chronic periodontitis were eligible for inclusion in this analysis. Full mouth periodontal probing along with peri-apical radiographs utilizing a paralleling technique was completed at a baseline appointment. No exclusions were made based on nicotine use, or medical history. None of these patients had been treated with adjunctive antibiotics. 7 patients were smokers, and 39 non-smokers. Informed consent for the use of an erbium laser in this procedure was obtained.

Patients with at least one angular intrabony defect, related to a probing depth of at least $5 \mathrm{~mm}$, received a combination of conventional root surface instrumentation, followed by an Er,Cr:YSGG laser application for a total of 79 defects. For the surgical procedure, $2 \%$ lidocaine hydrochloride solution with 1:80,000 adrenaline local anaesthetic was administered to achieve both tooth and soft tissue anaesthesia. Supra- and subgingival debridement was carried out using ultrasonic power driven instrumentation. The respective periodontal sites were treated with the Er.Cr:YSGG laser using a 14mm, 500 micron radial firing periodontal tip (RFPT5, Biolase, Irvine, CA, USA). The settings used were the following: power $1.5 \mathrm{~W}$, frequency $30 \mathrm{~Hz}, 20 \%$ water, $11 \%$ air, $\mathrm{H}$ (short pulse 60us) mode. The tip was inserted into the base of the pocket maintaining an angle parallel to the long axis of the root in sweeping motions both in a vertical and horizontal dimension until no granulation tissue was noted exiting the pocket, as shown in Figure 1.

Intrabony defects were managed by utilizing the radial firing periodontal laser tip as described above and inserted into the pocket until it touched osseous structure and was then withdrawn slightly. It was moved in a similar fashion with the same settings, until no further granulation tissue was noted as being flushed out of the pocket. A curette was used to degranulate along the bony walls of the defect. The laser tip was then re-inserted in the pocket, using the same setting except an altered frequency of $50 \mathrm{~Hz}$. Again, the tip was moved slowly, and angled parallel to the root surface, and then towards all the osseous levels surrounding the root. The tip was placed outside of the periodontal pocket parallel to the tissue to remove the epithelium surrounding the tooth by a distance of at least 5 millimeters from the gingival margin. This was seen visibly as white mottled tissue.

Patients were advised to commence normal brushing the following day post-operatively and to use the appropriate sized interdental brushes with no postoperative rinse or medicament. No occlusal adjustment was carried out and no adjunctive antibiotics were utilized.

Periodontal probing reassessment was performed at 5 to 8 months. Peri-apical radiographs (paralleling technique) were repeated on those sites with intrabony defects at the same visit.

\section{Operator, examiners, and exclusions}

The same periodontist (RAF) performed the initial charting and reassessment of periodontal pockets to the nearest millimeter ( $\mathrm{mm}$ ) using a $15 \mathrm{~mm}$ UNC probe. The radiographs taken before and after treatment were done using standardized film holders and radiographic units. The same respective operator performed all of the laser surgical procedures in private practice.

A second blinded examiner (RW) performed the radiographic assessment. Pre- and post-operative radiographs for each intrabony site were placed side by side on a black viewing background. Due to the nonstandardization of radiographs taken, gain in bone height was measured as a ratio of root length. This analysis of relative bone height within the defect was carried out by measuring root length from CEJ (or reproducible tooth coronal landmark as a restorative margin) to the most identified coronal extent of bone height on the root surface (shown in figure 2 ). Analysis of each pair was performed and recorded for statistical analysis.

A further 15 radiographs were available for analysis after more than 12 months, and the same measurements were repeated to measure both increase in lin- 
ear bone height and percentage bone fill (FJH).

\section{Results}

Patient demographics consisted of 46 patients and 79 total defects to undergo analysis. In all cases, healing was uneventful, with no post-operative infections, administration of antibiotics, or other adverse events. Some patients reported temperature sensitivity, mild soreness or discomfort on brushing, or use of analgesics for the first few days.

Pocket depth reductions of the intrabony defects are shown in Table 1. The mean probing depth before treatment was $8.1 \pm 1.9 \mathrm{~mm}$ (range 5 to $14 \mathrm{~mm}$ ) and following surgical laser therapy $2.4 \pm 0.9 \mathrm{~mm}$ (range 1 to $6 \mathrm{~mm}$ ) demonstrating significant reduction ( $\mathrm{p}$ $<0.001$ ). Figure 3 shows the mean probing depth for each patient before and after treatment.

Radiographic analysis of baseline and final images also demonstrated an improvement of osseous apposition from $1.1 \pm 0.4$ to $0.9 \pm 0.4$ units respectively as measured from the CEJ to the coronal aspect of the osseous levels. $(\mathrm{p}<0.001)$.

An additional analysis was performed of the radiographic findings to demonstrate a gain in bone height. The difference between baseline and post surgery (5-8 months later) provided the descriptive stats and 1 sample t-test to explore if this difference was different from zero. These measurements are a ratio, and do not have a value (e.g. millimeters). Therefore, one can multiply by 100 and demonstrate a gain in bone height by $19 \% \pm 28 \%(\mathrm{p}<0.001)$. More interestingly, despite the small increase in bone height, the bony infill of the defects visible radiographically is marked in all cases. (Figure 4).
From the measurements of the small sample of patients that had more than one year follow-up radiographs, in these sites there was a significant gain in linear bone height within intrabony defects $(18 \pm 11 \%$ of root length) but no change in suprabony bone height. 9 of the 15 sites showed 50\% or more bony infill of the intrabony defect (as shown in figure 6), with the serial radiographs showing continued bone fill over time (as shown in fig 7). One patient (number 7) showed an initial increase in bone fill within the defect in the first 5-8 months, followed by subsequent deterioration and loss of bone, which could not be explained clinically given that the pocket remained healed.

\section{Discussion}

Most previous studies reported on the use of lasers in periodontal therapy have mainly investigated diode, Erbium: Yttrium Aluminium Garnet (Er:YAG) and Nd:YAG lasers 5, 6, 10, 11, 12, 13). However, the Er,Cr:YSGG laser has had few studies in the field of periodontology, and on occasion has been grouped together with the Er:YAG laser due to having similar wavelengths and both having hard and soft tissue targets.

In previous clinical studies with Erbium lasers, the findings have varied both in efficacy and superiority over conventional scaling as a treatment modality. Several studies seem to imply the use of adjunctive laser treatment leads to a prolonged stability, with less bleeding on probing, higher CAL gain, and less recession one and two years post-operatively 8, 14). Conversely, no significant advantage was reported in other studies $\left.{ }^{4}, 10\right)$. The use of Er,Cr:YSGG has also been compared to conventional surgery (open flap

Table 1: Probing Depth and Radiographic Bone Height for Pre- and Post-operative (5-8 months later) assessment

\begin{tabular}{|c|c|c|c|c|}
\hline & $\begin{array}{l}\text { Pre-operative Probing } \\
\text { Depth }(\mathrm{mm})\end{array}$ & $\begin{array}{l}\text { Post-operative Probing } \\
\text { Depth }(\mathrm{mm})\end{array}$ & $\begin{array}{c}\text { Pre-operative Bone } \\
\text { Height }\end{array}$ & $\begin{array}{c}\text { Post-operative Bone } \\
\text { Height }\end{array}$ \\
\hline Mean \pm SD & $8.1 \pm 1.9$ & $2.4 \pm 0.9$ & $1.1 \pm 0.4$ & $0.9 \pm 0.4$ \\
\hline Median & 8.0 & 2.0 & 1.0 & 0.9 \\
\hline Interquartile Range & 2.0 & 1.0 & 0.6 & 0.6 \\
\hline Minimum & 5.0 & 1.0 & 0.3 & 0.2 \\
\hline Maximum & 14.0 & 6.0 & 2.7 & 2.3 \\
\hline $\begin{array}{c}\text { Wilcoxon Signed Ranks } \\
\text { Test }(\alpha=0.05)\end{array}$ & \multicolumn{2}{|c|}{$\mathrm{P} \leq .001$} & \multicolumn{2}{|c|}{$\mathrm{P} \leq .001$} \\
\hline
\end{tabular}




\section{ORIGINAL ARTICLES}
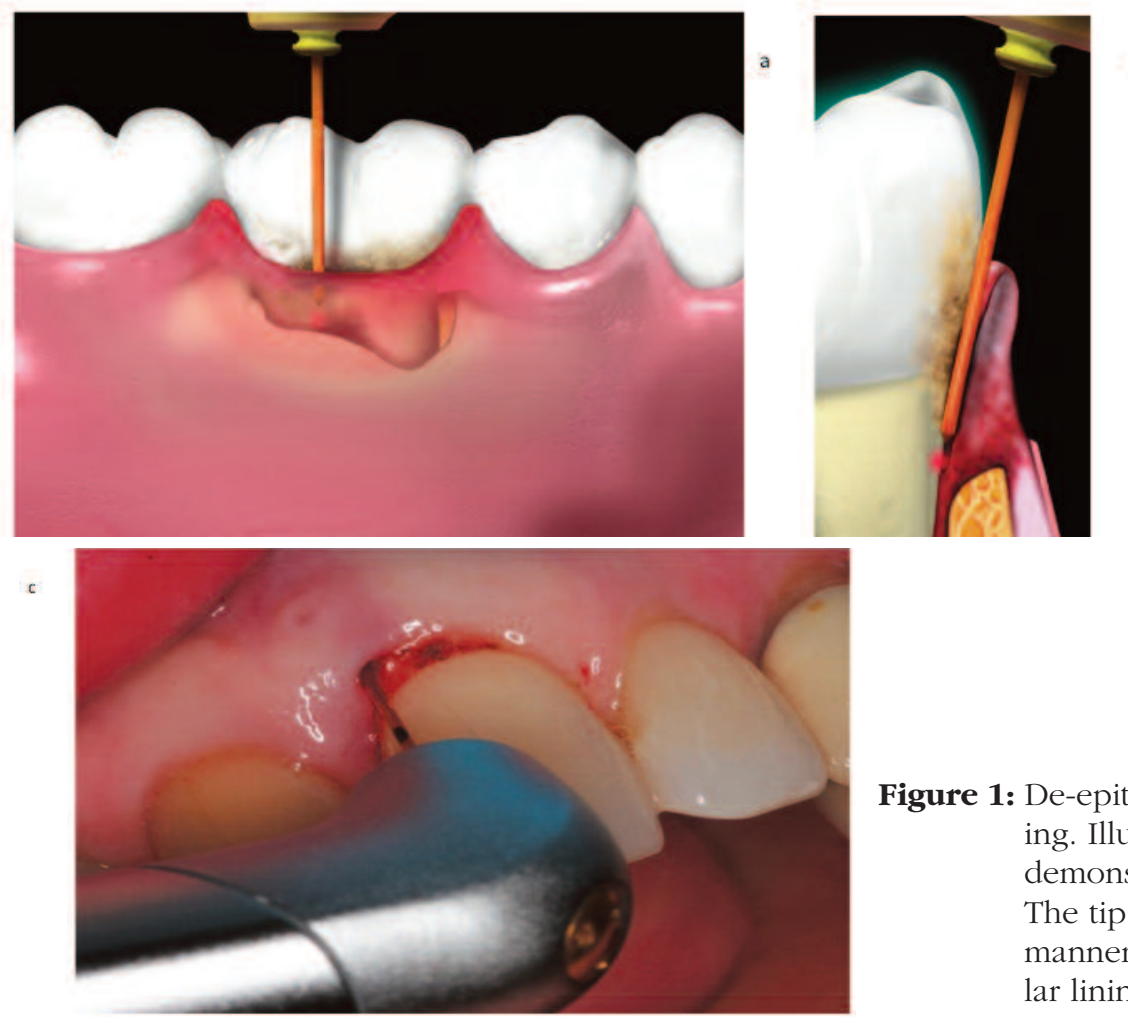

Figure 1: De-epithelialization of diseased sulcular lining. Illustrations (a,b) and clinical photo (c) demonstrating radial firing tip placement. The tip provides energy moving in a lateral manner with the target being both the sulcular lining and the root surface.
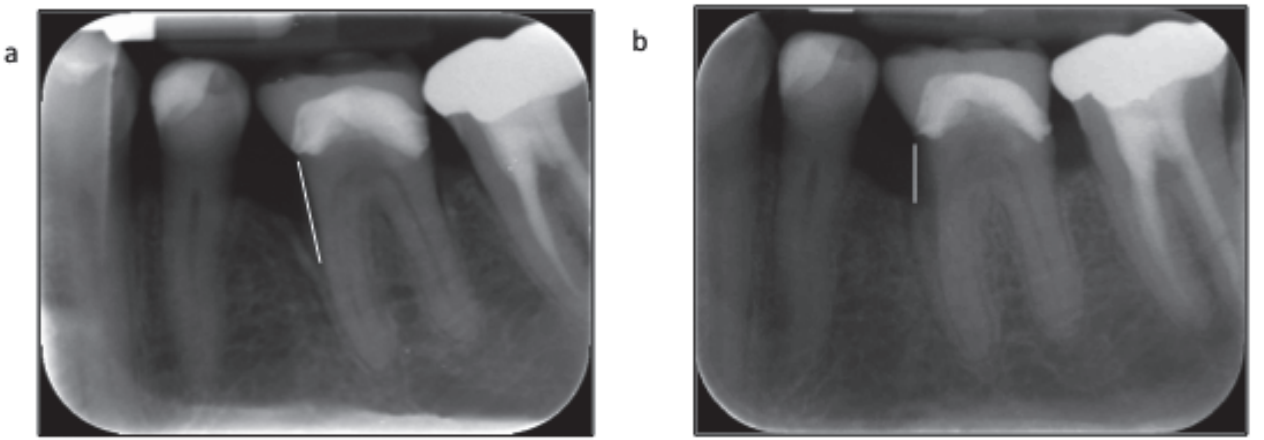

Figure 2: Measuring a fixed point to the most coronal osseous level at baseline (a) and the most coronal osseous level at the post laser surgery evaluation (b).

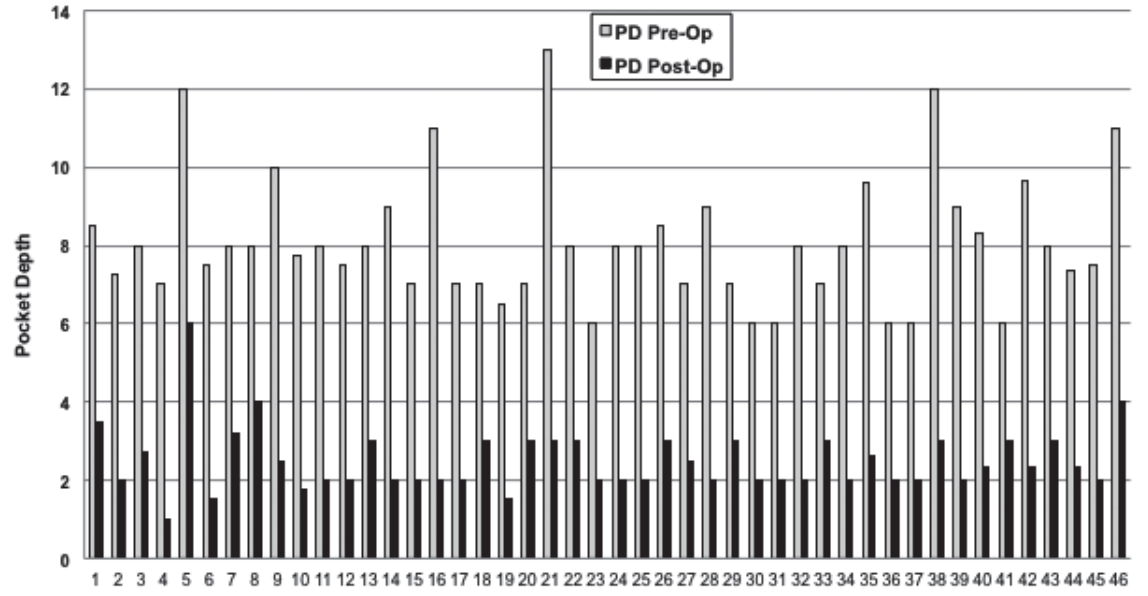

Figure 3: Mean pocket depth measurements $(\mathrm{mm})$ of infrabony pockets for all 46 patients pre- and post-operatively (5-8 months later). 


\section{ORIGINAL ARTICLES}

debridement) and found to achieve similar CAL gains, but with less gingival recession. ${ }^{9)}$

A systematic review by Karlsson et al., 15) based on studies that looked at the effectiveness of laser therapy as an adjunct to non-surgical periodontal treatment in subjects with chronic periodontitis was only able to use 25 abstracts and 4 randomised controlled clinical trials, all using different laser methods, and therefore making it impossible to conduct a meta-analysis. The conclusions to most studies suggest the need for more
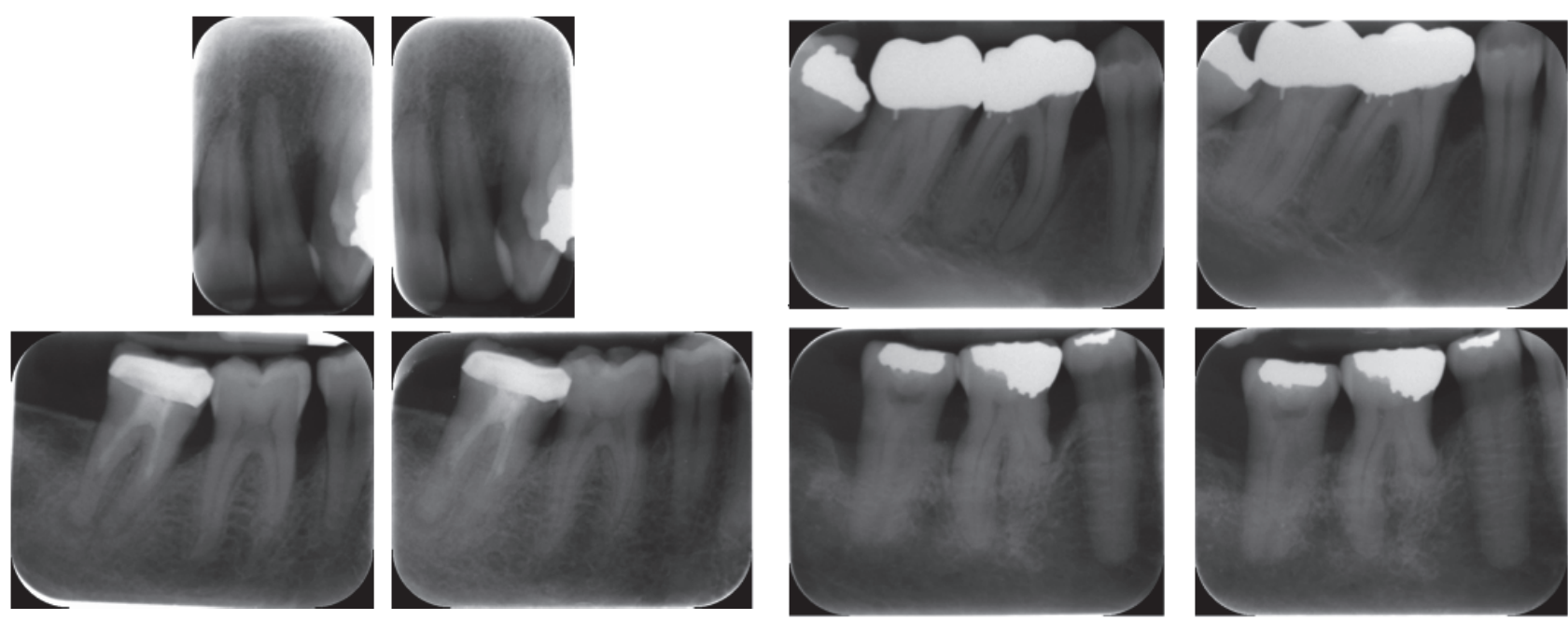

Figure 4: Paired radiographs pre-op (on left) and post-op (on right) showing evidence of radiographic osseous fill of intra-bony defects 8 months following treatment.

a

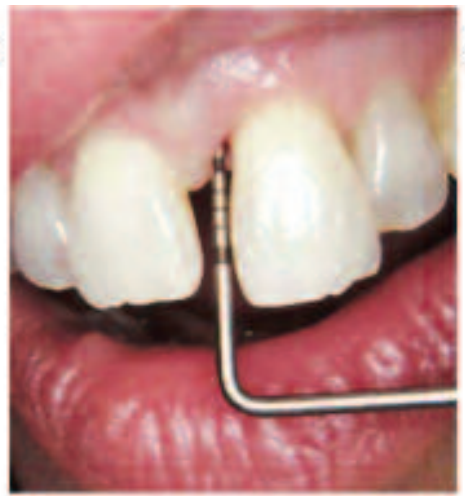

b
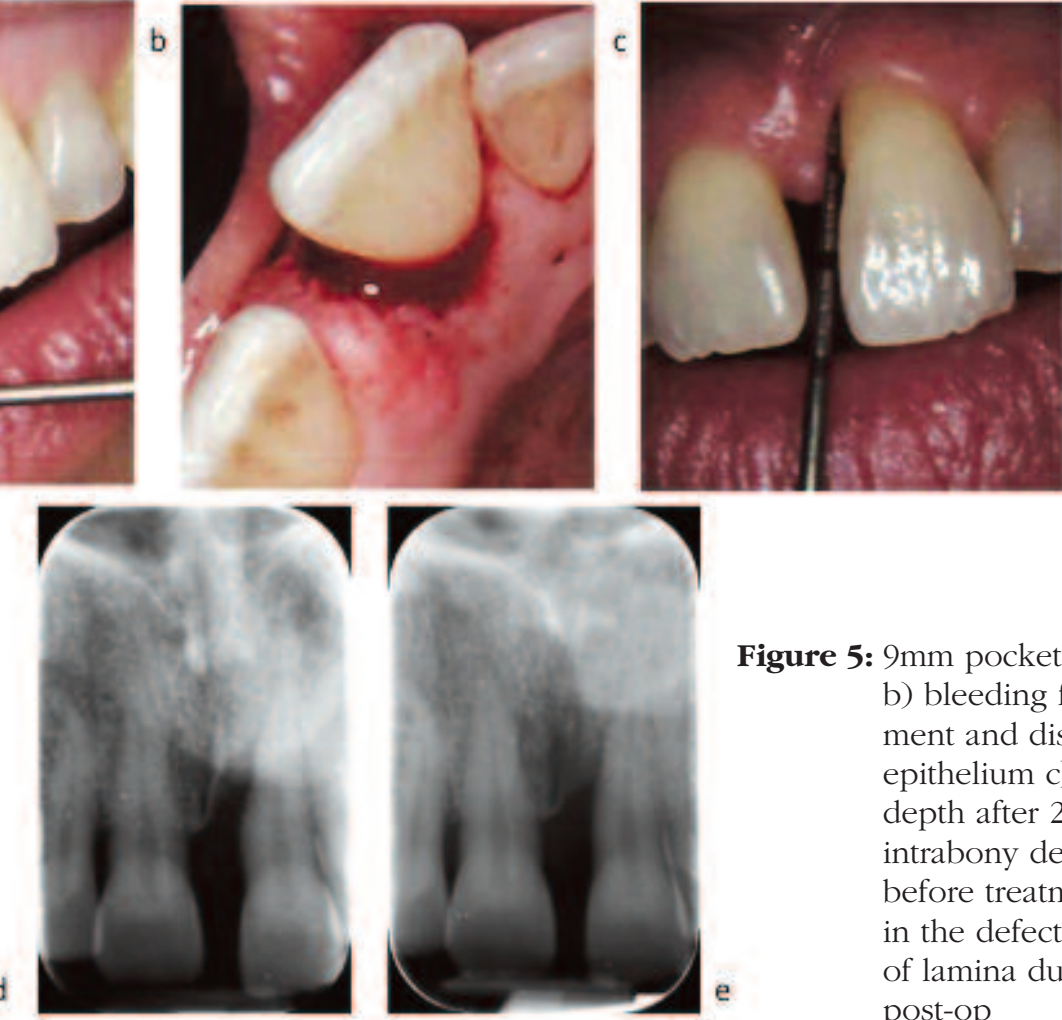

Figure 5: $9 \mathrm{~mm}$ pocket before treatment b) bleeding following treatment and disrupted outer epithelium c) $2 \mathrm{~mm}$ probing depth after 2 months d) wide intrabony defect on mesial before treatment e) bone fill in the defect and formation of lamina dura 8 months post-op 


\section{ORIGINAL ARTICLES}

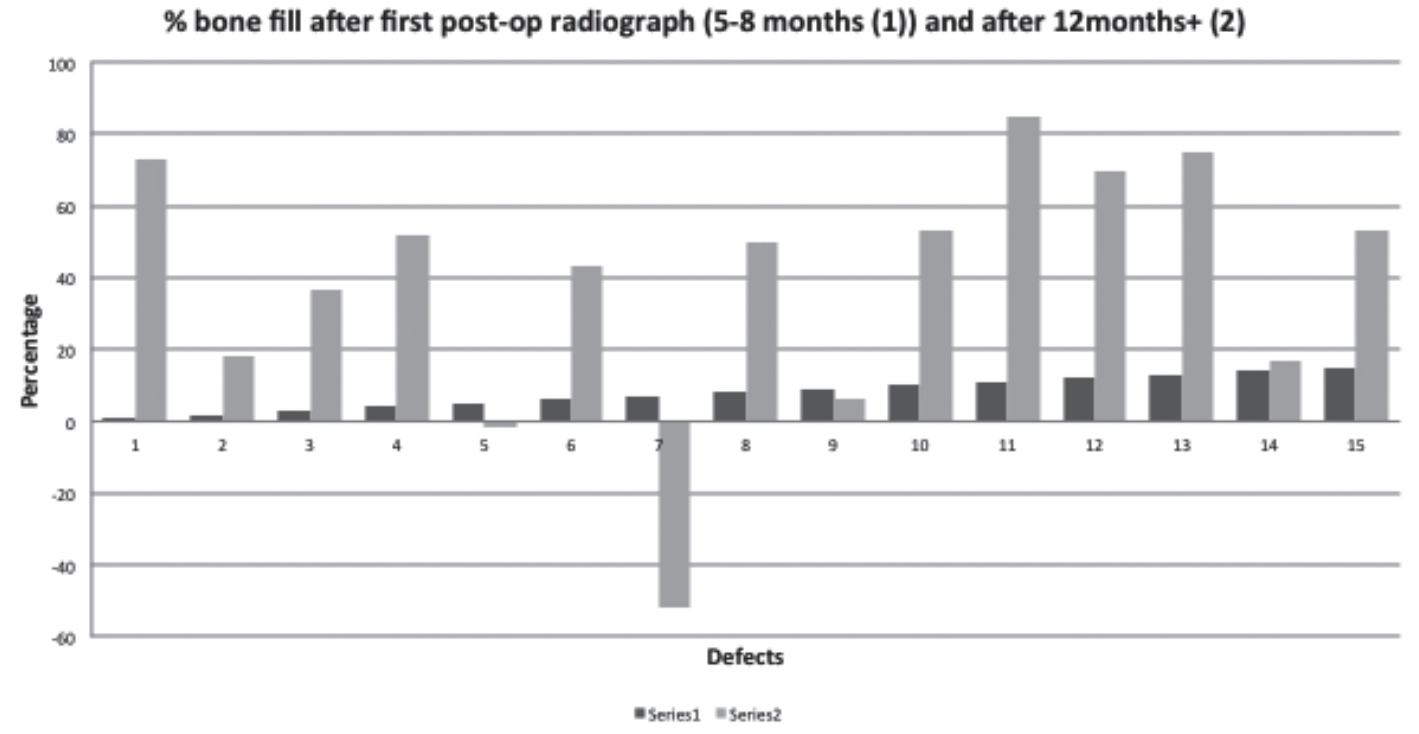

Figure 6: Percentage bone fill in each defect at first post-op of 5-8 months (1) and subsequent post-op $>12$ months later (2)
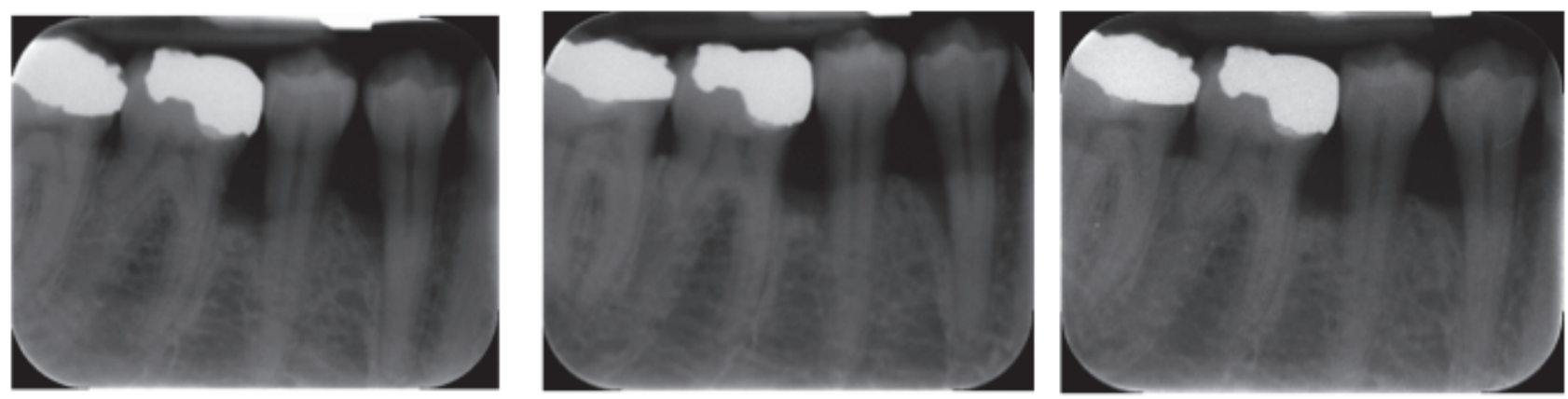

Figure 7: Serial radiographs of one case taken at baseline, 8 months later and 18 months later, showing continued bone fill in the defects over time.

clinical trials. Individual reports are of similar results to traditional methods, but until there is a larger science base, this can only be termed anecdotal.

The possible reasons why adjunctive flapless laser therapy might result in improved outcomes similar to conventional therapy might include improved root surface debridement and removal of pocket granulation tissue. Studies in vitro have reported that the Er,Cr:YSGG laser is effective in the removal of biofilm, smear layer, root surface endotoxins, infected cementum and calculus 16, 17, 18). Some studies have found this laser to be as effective as manual and power scalers, without causing damage to the root surface, both in terms of morphological changes such as cracks and thermal damage as melting or carbonisation. It has been postulated that the removal of the epithelial lining, as advocated in the manufacturer's protocol may also have a role to play in the effectiveness seen by delaying epithelial down-growth during pocket healing 9). Of course, without histological evidence, one cannot confirm if all the epithelium is removed, and hence described as a disruption in the epithelium in this study. Animal studies have also suggested that the Er,Cr:YSGG laser energy may also have a direct stimulatory effect on bone healing through gene regulation and cytokine production ${ }^{19)}$. All of these actions could perhaps be plausible mechanisms for the changes seen in this extended case series. Aoki ${ }^{20)}$ describes a mini- 
mally invasive surgical technique called laser-assisted comprehensive periodontal pocket therapy (LCPT), using an Er:YAG laser. This protocol has similar steps to the ones used here, including use of the laser to remove outer epithelium, pocket epithelial lining and diseased connective tissue, down to bone, with the use of a curette on bone to aid this latter stage; laser-modification of the root surface, along with detoxification, removal of smear layer and calculus; stabilisation of a blood clot, and some low level laser effects resulting in biostimulation 21, 22, 23). Likewise, similar steps, incorporating laser-tissue interaction on the soft tissue are relayed in the laser assisted new attachment protocol (LANAP), using an Nd:YAG laser. This procedure also involves a first laser pass to remove inflamed pocket epithelium, while the calculus removal and bone decortication is carried out with other instruments. A second laser pass is then carried out which helps to stabilise the blood clot, and due to the more deeply penetrating effects of the Nd:YAG, the biostimulatory effect is one of the plausible mechanisms for the regeneration seen using this protocol $1,2,3$ ).

In recent periodontal regeneration studies there has been great emphasis on the importance of wound stability and indeed the use of minimally-invasive surgical techniques using mini-flaps has been shown to result in equivalent regenerative outcomes even without the application of grafts and other regenerative materials $24,25,26)$. In the use of the laser described here, debridement and granulation tissue removal is carried out using a minimally invasive surgical technique, without raising a flap, assuming adequate wound debridement ${ }^{14)}$

The issues that seem to arise are that lasers have different wavelengths, might work through different mechanisms, use different tips for different procedures at different angles, and have countless different settings applicable, so it becomes very difficult to accumulate a good evidence base on best practice. One of the key requirements for future studies of laser applications is to identify the optimal parameters, which can profoundly influence therapeutic outcomes ${ }^{27)}$. One of

\section{References}

1: Yukna R A, Carr R L, Evans G H. (2007): Histologic evaluation of an Nd:YAG laser-assisted new attachment procedure in humans. Int $\mathrm{J}$ Periodontics Restorative Dent 27: 577-587.

2: Nevins M L, Camelo M, Schupbach P, Kim S W, Kim D M, Nevins M (2012): Human clinical and these parameters that should be included is patientrelated outcomes ${ }^{28}$ ). The hypothesis that can be considered is that both traditional flap procedures and laser closed flap procedures may provide equivalent clinical results but closed flap procedures provide more patient acceptable outcomes as level of discomfort and reliance on analgesics.

As a retrospective analysis of an extended case series these results should be considered a low level of evidence for efficacy of adjunctive surgical laser treatment, but the outcomes are sufficient to warrant the need for proper randomised controlled studies of the Er,Cr:YSGG laser in the surgical and non-surgical management of periodontitis. An additional shortcoming of the retrospective study is not measuring clinical attachment levels thus some of the resulting improvement in pocket depth could also be from the respective recession, but unfortunately this data was not available for the whole group of patients. A radiographic stent could have been utilized to accurately measure change instead of paired non-standardized films. However, the films were analysed by a blinded examiner. These studies of measuring visible osseous levels can not take into consideration a level of increased radiopacity as can occur with subtraction radiography thus undermining a notation of true osseous fill and maturation. Moreover, the analysis may have demonstrated more osseous fill if the study had been carried out longer than the 5 to 8 month time frame, which was the case with a small sample of the patients.

This retrospective case series suggests that the use of the Er,Cr:YSGG laser for surgical treatment of periodontal pockets may be an effective adjunctive management for infrabony defects associated with chronic periodontitis. In future studies, there is a need to identify optimal protocols and parameters for use of Er,Cr:YSGG lasers in specific applications as these may profoundly influence therapeutic responses ${ }^{27)}$. In particular, further well-designed RCTs are required to investigate these findings under more rigorous test conditions.

histologic evaluation of laser- assisted new attachment procedure. Int J Periodontics Restorative Dent 32:497-507.

3: Nevins M, Kim S W, Camelo M, Martin I S, Kim D, Nevins M (2014): A prospective 9-month human clinical evaluation of Laser-Assisted New 
Attachment Procedure (LANAP) therapy. Int J Periodontics Restorative Dent 34(1):21-27. doi: 10.11607/prd.1848.

4: Schwarz F, Aoki A, Becker J, Sculean A (2008): Laser application in non-surgical periodontal therapy: a systematic review. J Clin Periodontol 35:2944.

5: Rotundo R, Nieri M, Cairo F, Franceschi D, Mervelt J, Bonaccini D, Esposito M, Pini-Prato G (2010): Lack of adjunctive benefit of Er:YAG laser in nonsurgical periodontal treatment: a randomized splitmouth clinical trial. J Clin Periodontol 37: 526-533.

6: Sgolastra F, Petrucci A, Gatto R, Monaco A (2012): Efficacy of Er:YAG laser in the treatment of chronic periodontitis: systematic review and meta-analysis. Lasers Med Sci 27: 661-673.

7: Sgolastra F, Severino M, Petrucci A, Gatto R, Monaco A (2014): Nd:YAG laser as an adjunctive treatment to nonsurgical periodontal therapy : A meta-analysis. Lasers Med Sci 29(3): 887-895

8: Kelbauskiene S, Baseviciene N, Goharkhay K, Moritz A, Machiulskiene V (2011): One-year clinical results of Er,Cr:YSGG laser application in addition to scaling and root planing in patients with early to moderate periodontitis. Lasers Med Sci 26: 445-452.

9: Gupta M, Lamba A K, Verma M, Faraz F, Tandon S, Chawla K and Koli D K (2013): Comparison of periodontal open flap debridement versus closed debridement with Er,Cr:YSGG laser. Australian Dental Journal 58: 41- 49

10: Sculean A, Schwarz F, Berakdar M, Windisch P, Arweiler N B \& Romanos G E (2004): Healing of intrabony defects following surgical treatment with or without an Er:YAG laser. J Clin Periodontol 31: 604-608.

11: Slot D E, Kranendonk A A, Paraskevas S. \& Van der Weijden F (2009): The effect of a pulsed Nd:YAG laser in non-surgical periodontal therapy. J Periodontol 80: 1041-1056.

12: Thomas M. \& Shafer K (2011): Insufficient evidence that pulsed Nd:YAG laser treatment is superior to conventional nonsurgical therapy in the treatment of periodontal disease. J Am Dent Assoc 142: 194-195.

13: Slot D E, Timmerman M F, Versteeg P A, van der Velden U, van der Weijden F A (2012): Adjunctive clinical effect of a water-cooled Nd:YAG laser in a periodontal maintenance care programme: a randomized controlled trial. J Clin Periodontol 39: 1159-1165.

14: Dyer B, Sung E C (2012): Minimally Invasive
Periodontal Treatment Using the Er,Cr: YSGG Laser. A 2-year Retrospective Preliminary Clinical Study. Open Dent J 6: 74-78.

15: Karlsson M R, Diogo Lofgren C I, Jansson H M (2008): The effect of laser therapy as an adjunct to non-surgical periodontal treatment in subjects with chronic periodontitis: a systematic review. J Periodontol 79: 2021-2028.

16: Ting C, Fukuda M, Watanabe T, Aoki T, Sanaoka A, Noguchi T (2007): Effects of Er,Cr:YSGG laser irradiation on the root surface: morphologic analysis and efficiency of calculus removal. J Periodontol 78: 2156-2164.

17: Noori Z T, Fekrazad R, Eslami B, Etemadi A, Khosravi S, Mir M (2008): Comparing the effects of root surface scaling with ultrasound instruments and Er,Cr:YSGG laser. Lasers Med Sci 23: 283-287.

18: Hakki S S, Korkusuz P, Berk G, Dundar N, Saglam M, Bozkurt B, Purali N (2010): Comparison of Er,Cr:YSGG laser and hand instrumentation on the attachment of periodontal ligament fibroblasts to periodontally diseased root surfaces: an in vitro study. J Periodontol 81: 1216-1225.

19: Pavone C, Perussi LR, de Oliveira GJ, Scardueli CR, Cirelli JA, Cerri PS, Junior EM, Spolidorio LC, Marcantonio RA (2015): Effect of Er,Cr:YSGG laser application in the treatment of experimental periodontitis. Lasers Med Sci 30:993-999

20: Aoki A, Mizutani K, Schwarz F et al. (2015): Periodontal and peri-implant wound healing following laser therapy. Periodontol 2000 68: 217-269

21: Pourzarandian A, Watanabe H, Ruwanpura SM, Aoki A, Ishikawa I (2005): Effect of low-level Er:YAG laser irradiation on cultured human gingival fibroblasts. J Periodontol 76: 187-193.

22: Pourzarandian A, Watanabe H, Ruwanpura SM, Aoki A, Noguchi K, Ishikawa I (2005): Er:YAG laser irradiation increases prostaglandin E production via the induction of cyclooxy- genase-2 mRNA in human gingival fibroblasts. J Periodontol Res 40: 182-186.

23: Ogita M, Tsuchida S, Aoki A, Satoh S, Sawabe M, Nanbara H, Kobayashi H, Takeuchi Y, Mizutani K, Sasaki Y, Nomura F, Izumi Y (2015): Increased call proliferation and differential protein expression induced by low-level Er:YAG laser irradiation in human gingival fibroblasts:proteomic analysis. Lasers Med Sci 30:1855-66

24: Trombelli L, Farina R (2008): Clinical outcomes with bioactive agents alone or in combination with grafting or guided tissue regeneration. J Clin Periodontol 35: 117-135. 
25: Trombelli L, Simonelli A, Pramstraller M, Wikesjo U M, Farina R (2010): Single flap approach with and without guided tissue regeneration and a hydroxyapatite biomaterial in the management of intraosseous periodontal defects. J Periodontol 81: 1256-1263.

26: Cortellini P, Tonetti M S (2011): Clinical and radiographic outcomes of the modified minimally invasive surgical technique with and without regenerative materials: a randomized-controlled trial in intra-bony defects. J Clin Periodontol 38: 365-373.

27: Kotsakis G A., Konstantinidis I, Karoussis I K, Ma X, Chu H (2014): A Systematic Review and MetaAnalysis of the Effect of Various Laser Wavelengths in the Treatment of Peri-Implantitis. J Periodontol 10:1902/jop.2014.130610

28: McGuire M K, Scheyer T and Gwaltney C (2014): Commentary: Incorporating patient-reported outcomes in periodontal clinical trials. J Periodontol 85: 1313-1319 